\title{
Ön çapraz bağ yaralanması tedavisinin komplikasyonları
}

\section{Complications of the treatment of anterior cruciate ligament injuries}

\author{
Ömer Faruk Taşer, Berkin Toker
}

Acıbadem Fulya Hastanesi, Ortopedi ve Travmatoloji Bölümü, İstanbul

\begin{abstract}
Ön çapraz bağ (ÖÇB) yaralanmalarında cerrahi tedavi sıklıkla ve başarı ile uygulanmaktadır. Basit bir menisküs cerrahisindeki komplikasyon oranı \%1,5 iken, ÖÇB cerrahisi sonrası bu oran \%9'lara kadar çıkmaktadır. Komplikasyonlar ile başa çıkabilmek için risk faktörlerini iyi ortaya koymak gerekir. Komplikasyonları; yaralanmanın boyutu, ameliyat öncesi faktörler, ameliyat dönemi teknik faktörler ve ameliyat sonrası dönem ve rehabilitasyon olarak bölümlere ayırarak incelemek daha doğru olacaktır. Özellikle artrofibrozis ve enfeksiyon, tedavilerinin zor ve uzun sürmesi özellikleri ile ÖÇB cerrahisi sonrası cerrahı ve hastayı uğraştıran komplikasyonlardandır. Artrofibrozisin başlıca nedenleri arasında ameliyat öncesi diz eklem hareket açıklığı (EHA)'nın yetersiz olması, cerrahideki teknik hatalar, eşlik eden iç yan bağ (IYB) rekonstrüksiyonu, enfeksiyon, immobilizasyon sayılabilir. Bazen de risk faktörleri olmadan artrofibrozis geliştiği bilinmektedir. Vücudun geliştirdiği immün sistem aracılığı ile salınan bazı mediatörler bu reaksiyondan sorumlu tutulmaktadır. Bir diğer korkulan komplikasyon ise eklem enfeksiyonu, yani ameliyat sonrası gelişen septik artrittir. Enfeksiyondan şüphelenildiği anda uyanık davranılıp başlanılan kültür spesifik antibiyoterapi ve erken yapılan artroskopik debridman ya da debridmanlar tedavide çok önemlidir.
\end{abstract}

Anahtar sözcükler: ön çapraz bağ; artrofibrozis; septik artrit; enfeksiyon; komplikasyon

\begin{abstract}
Surgical treatment of anterior cruciate ligament (ACL) injuries are often performed with high success rates. While complication rates for a simple meniscus surgery stands at $1.5 \%$, the rates increase up to $9 \%$ after ACL surgeries. To tackle possible complications, the risk factors have to be identified and dealt with. We will divide the risk factors into four groups, namely the severity of the injury, preoperative factors, perioperative factors and postoperative period and rehabilitation. Arthrofibrosis and infection in particular are the most troublesome complications resulting in difficult and long lasting treatment periods. It is well known that risk factors of arthrofibrosis include; preoperative limited ROM, surgical technical faults, additional collateral ligament injuries, infection and immobilization. At times arthrofibrosis can occur without any obvious risk factors, which can be associated with mediators produced as an immune system response. Another troublesome complication is septic arthritis. It is very essential to be at an alarm in suspected cases. Culture specific antibiotherapy and arthroscopic debridement and irrigation/irrigations are the main treatment options in deep joint infections.
\end{abstract}

Key words: anterior cruciate ligament; arthrofibrosis; septic arthritis; infection; complication

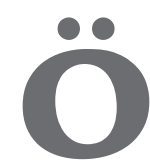

n çapraz bağ (ÖÇB) yaralanması son yıllarda giderek artan sayıda ve birçok merkezde yapılır hale gelmiştir. Amerika'da her yıl ortalama 150.000 civarında ÖÇB yaralanması ile karşılaşılıp bunların 95.000'i cerrahi tedavi almaktadır. Bu kadar yaygın yapılan bir cerrahinin de komplikasyonları sıklıkla karşımıza çıkmaktadır. ${ }^{[1]}$ Bir menisküs cerrahisi sonrası ortalama komplikasyon oranı \%1,5 iken ÖÇB cerrahisi sonrası bu oran \%9'lara kadar çıkmaktadır. ${ }^{[2]}$
ÖÇB cerrahisi sonrası komplikasyonları anlatırken bu komplikasyonların ortaya çıkmasına neden olan risk faktörlerini bölümlere ayırarak incelemek daha doğru olacaktır.

- Yaralanmanın boyutu

- Ameliyat öncesi faktörler

- Ameliyat dönemi teknik faktörler

- Ameliyat sonrası dönem ve rehabilitasyon

- İletişim adresi: Dr. Berkin Toker, Acıbadem Fulya Hastanesi, Hakkı Yeten Cad., Yeşilçimen Sk., no: 23. Fulya, İstanbul Tel: 0532 -3209691 e-posta: mberkintoker@hotmail.com

- Geliş tarihi: 28 Mayıs $2020 \quad$ Kabul tarihi: 1 Haziran 2020 


\section{YARALANMANIN BOYUTU}

Yüksek enerjili yaralanmalar, dizde ÖÇB yırtı̆̆ı yanında hematom, kas zedelenmesi, ve değişik boyutlarda yumuşak doku veya kemik hasarı ile birlikte olabilir. Yaralanmanın boyutu arttıkça bu hastaların ameliyat sonrası dönemde hareket kısıtlılığı problemi ile karşımıza gelme ihtimali yükselmektedir. Özellikle ÖÇB yaralanmasına menisküs ile diz eklem içi ve dışı diğer bağların da hasarının eklenmesi bir taraftan tedavinin zamanlamasını ve boyutunu değiştirirken diğer taraftan muhtemel cerrahi süresini uzatmaktadır. Yapılan çoklu cerrahi işlemler sonrası immobilizasyon sürelerinin uzayabilmesi özellikle eklem hareket açıklığı (EHA) kısıtlıığına yol açabilir. ${ }^{[3]}$ Çoklu bağ yaralanmalarında özellikle lateral ve posterolateral köşe lezyonlarında, tamir edilebilecekse erken dönemde operasyon önerilmektedir. Erken dönemde yapılamayacak olgularda bile erken EHA'nın başlanması diz çevresinde skar dokusu gelişmemesi adına önemlidir.

Çoklu bağ yaralanmalarında cerrahi tedavi sonrası enfeksiyon riskinin standart bir ÖÇB cerrahisine oranla daha yüksek olduğu akılda tutulmalıdır.

\section{AMELIYAT ÖNCESI FAKTÖRLER}

ÖÇB cerrahisini yaralanmadan hemen sonra diz hareket kısıtlılığı ve ödem mevcut iken yapmak ameliyat sonrası komplikasyon riskini artırmaktadır.

Shelbourne ve ark., erken yapılan olgularda artmış artrofibrozis oranları bildirmişlerdir. ${ }^{[4]}$ Benzer bir çalışmada Wasilewski ve ark., akut ÖÇB cerrahisi sonrası \%22 oranında artrofibrozis bildirmişlerdir. ${ }^{[3]}$ Harner ve ark. ise ilk bir ay içinde yapılan olgularda hareket açıklığı problemi yaşayan hasta oranını \%37 olarak bulmuşlardır. ${ }^{[5]}$ Bu yayınlara karşılık Bach ve ark., erken ve geç dönemde yapılan olgularda diz hareket açıklığı açısından fark saptamasalar da "erken cerrahinin bir avantajı olmadığı" vurgusunu yapmışlardır. ${ }^{[6]}$ Literatür bilgisinin yanında bizim kendi tecrübelerimize göre de ÖÇB cerrahisi öncesi menisküs yırtığı, yan bağ yaralanması vb. ek majör bir patoloji yoksa diz hareket açıklığının 0-120, kuadriseps kuvvetinin tam ve ödemin geçmiş olması ameliyat sonrası rehabilitasyon açısından ciddi bir avantaj sağlayacaktır.

\section{ARTROFIBROZis}

Diz ekleminde ÖÇB cerrahisi sonrası ortaya çıkan ağrılı hareket kısıtlıı̆ı̆ı artrofibrozis olarak adlandırılır. Artrofibrozis klinik bir tanıdır; ağrı, hareket kısıtlığı ve eklemde sertlik olarak üç klinik komponenti vardır. ${ }^{[7]}$

ÖÇB yaralanması ve/veya cerrahisi sonrası artrofibrozis geliştiğinin fark edilememesi rehabilitasyon sürecinde ciddi problemler doğuracaktır.
Artrofibrozisin primer ve sekonder olmak üzere iki temel formu vardır. ${ }^{[8]}$ Lokalize form veya benign form diye adlandırılan sekonder artrofibroziste; greftin yanlış yerleşimi, infrapatellar kontraktür sendromu ve patella infera, siklops sendromu, eşlik eden iç yan bağ lezyonu ve kötü programlanmış rehabilitasyon gibi altta yatan bir neden vardır. Buna karşın primer artrofibroziste masif bir fibrozis vardır. Burada lokalize formdan farklı olarak aktif ve proliferatif bir fibrozis söz konusudur. Bu nedenle primer artrofibrozis, jeneralize form olarak da adlandırılır. Primer artrofibroziste masif fibrozis yanında, dirençli (persistan) bir sinovit ve kronik bir enflamatuvar reaksiyon söz konusudur. ${ }^{[8]}$

Artrofibroziste diz hareket açıklığına göre bir sınıflama kullanılır. İlk olarak 1996 yılında Shelbourne tarafindan tarif edilen sınıflama[4], 2018 yılında Scott ve Shelbourne tarafından revize edilmiştir (Tablo 1). ${ }^{\left[{ }^{[8]}\right.}$

Artrofibroziste ekstansiyon kısıtlılığına posterior kapsüldeki skar dokusu, adhezyonlar ve siklops gelişimi neden olabilir. Fleksiyon kısıtlılığı ise kuadriseps kasındaki kısalığa, patella etrafında gelişen skar dokusuna, medial ve lateral kapsül komşuluğundaki yapışıklıklara bağlı olabilir.

Tip 1 artrofibroziste, aktivite ile ortaya çıkan bir diz önü ağrısı vardır. Eklem pasif olarak forse edildiğinde tam ekstansiyona gelir ama bırakıldığı zaman fleksiyon pozisyonuna geri döner, posterior kapsül kontraksiyonu bu tabloya katkıda bulunabilir. Tip 1 artrofibrozisin tipik örneği siklops lezyonudur (Şekil 1) ve tedavisi siklops nodülünün artroskopik olarak eksizyonundan ibarettir.

Tip II artrofibroziste, anteriorda mekanik blok varlığı veya ÖÇB greftinin noç (notch) içinde uyumsuzluğu yanında posterior kapsülde giderek sekonder gerginlik olması eklemin pasif olarak forse edildiğinde bile tam ekstansiyona gelmesine engel olur. Tip II artrofibrozisin tedavisi artroskopik olarak yapılabilir ve anterior nedbe dokusunun ve fibrotik Hoffa'nın eksizyonu yanında, diz tam ekstansiyonda greftin noç tavanına sıkışması varsa, noçplasti gerektirir.

Tablo 1. Artrofibrozisin klinik sınıflaması ${ }^{[8]}$

\begin{tabular}{|c|c|}
\hline Tip 1 & $<10^{\circ}$ ekstansiyon kısıtlılı̆̆ı, normal fleksiyon \\
\hline Tip 2 & $>10^{\circ}$ ekstansiyon kısıtlılığı, normal fleksiyon \\
\hline Tip 3 & $>10^{\circ}$ ekstansiyon kısıtlılığı, $>25^{\circ}$ fleksiyon kaybı \\
\hline Tip 4 & $\begin{array}{c}>10^{\circ} \text { ekstansiyon kısıtlılığı, }>30^{\circ} \text { fleksiyon kaybı } \\
\text { (ek olarak patella infera mevcuttur) }\end{array}$ \\
\hline
\end{tabular}




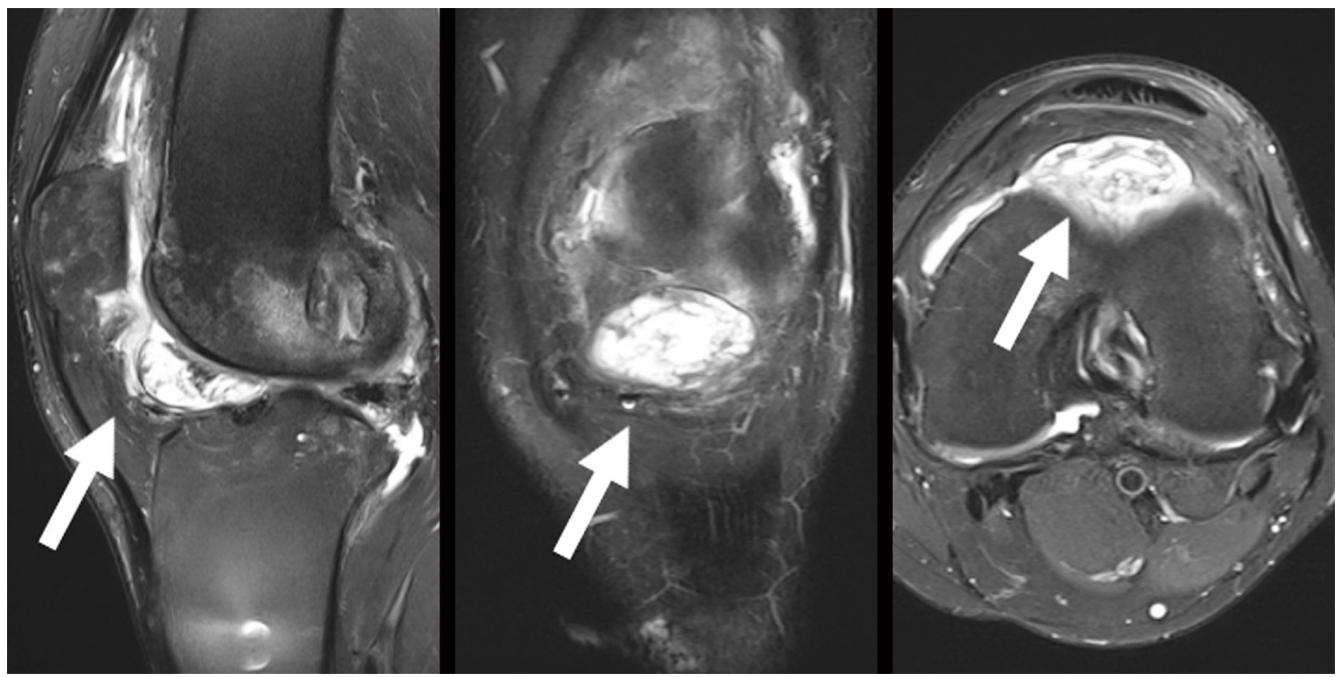

Şekil 1. Otuz üç yaş erkek hasta, ÖÇB rekonstrüksiyonu cerrahisi sonrası ameliyat sonrası 4. ayda diz ekstansiyon kısıtlılığı ile başvurdu. Çekilen manyetik rezonans görüntülerinde dizin anteriorunda yer alan ve ekstansiyonda noç tavanına sıkışan nodüler yapıda siklops lezyonu (beyaz ok).

Tip III artrofibroziste patellar mobilizasyonda azalma yanında, medial ve lateral kapsüler yapılarda gerginlik vardır. Tip III artrofibrozis tedavisinde, patellar tendon ile tibia arasındaki fibrotik Hoffa yağ dokusunun tamamen çıkarılır ve patella ve patellar tendonu tamamen serbestleştirebilmek için fibrotik kapsül vastus medialis oblikus ve vastus lateralis yapışma yerine kadar eksize edilir, gerekirse noçplasti eklenir ve eklem anestezi altında manipüle edilir.

Tip IV artrofibroziste eklem hareket açıklığı Tip III ile aynıdır, farkı tabloya patella inferanın eklenmiş olmasıdır. Tedavisinde Tip III artrofibrozis benzeri cerrahi işlemler yanında posteromedial insizyon ile posterior kapsülün eklem içinden güvenli bir şekilde femurdan sıyrılması gerekir. Tam ekstansiyon için tibianın $10^{\circ}$ dışa rotasyon hareketi gerektiğinden kapsülün sıyrılması için öncelikle postero-medial insizyon tercih edilmelidir.

Artrofibrozisten korunmak için öncelikle artrofibrozis gelişimini kolaylaştırıcı risk faktörlerini bilmek gerekir (Tablo 2). ${ }^{[1]}$ Çok tartışılan konulardan bir tanesi, bir ÖÇB yaralanması sonrası cerrahinin zamanlamasıdır ama burada esas dikkat edilmesi gereken husus, rekonstrüksiyon sırasında dizin fiziki kondisyonunun, travma ile cerrahi arasında geçen süreden çok daha önemli olduğudur. Uygun ameliyat zamanı için dizde effüzyon olmaması, EHA'nın tam, kuadriseps kontrolünün iyi ve kas kuvvetinin yeterli olması idealdir.

Aktif enflamasyonun devam ettiği veya tam hareket açıklığının kazanılamadığı dizlerde artrofibrozis riski ve insidansı daha yüksektir. Bu nedenle şişliğin ve enflamasyonun devam ettiği bir dizde akut cerrahiden kaçınılmalıdır. Proksimal femoral yapışma yerinden avülse olan veya mid-substans iç yan bağ yırtıkları esneklik kaybı (stiffness) ile iyileşme eğilimindedir; bu olgularda ÖÇB rekonstrüksiyonu öncesi tam EHA kazanılması önemlidir.

Ameliyat sonrası dönemde kuadriseps inhibisyonu oluşursa, hamstring tendonları dizi fleksiyona çeker ve hastalar patellar tendonu yeterince geremez. Kuadriseps inhibisyonu önlenemez veya süratle geri kazanılamazsa oluşan patellar tendon kontraktürü, patella inferaya yol açar.

Fleksiyon ve düz bacak kaldırma gibi kuadriseps kontrol egzersizleri patellar tendonu gerer ve tendonun kontrakte olmasına engel olur. Bu nedenle patella inferanın önlenmesi için ameliyat sonrası dönemde kuadriseps kontrol ve patellar tendon germe egzersizlerine özel önem verilmelidir.

Tablo 2. Artrofibrozis gelişimini kolaylaştırıcı risk faktörleri ${ }^{[1]}$

- Yaralanmanın büyüklüğü (diz çıkı̆ı, çoklu bağ yaralanması gibi...)

- Ameliyat öncesi diz EHA'nın yetersiz olması

- Cerrahideki teknik hatalar

- Eşlik eden iç yan bağ rekonstrüksiyonu

- Enfeksiyon

- Immobilizasyon

- Kronik diz effüzyonu, kronik sinovit

- Siklops lezyonu

- Refleks sempatik distrofi 
Hem hasta, hem de doktor-fizyoterapist açısından tedavisi çok zor olan primer (jeneralize) artrofibroziste yakın gelecekte fibrotik doku oluşmasını engelleyecek kimyasal ajanlar kullanılması tedavi açısından dönüm noktası olabilir. ${ }^{[9]}$

Artrofibrozis tedavisinde kritik olan nokta, artrofibrozis tanısı konulan hastanın fizik tedavi ile aşırı zorlanmaması gerektiğidir. Agresif tedavi genelde istenenin tersine, vücudun verdiği tepkiyi artırmaktadır. Illk hedef öncelikle ekstansiyonu sağlamak olmalıdır. Bu aşamada patellar mobilizasyon, gerektiğinde hastanın kendisine de öğretilerek düzenli yapılmalıdır. Ekstansiyonun kazanılması, yüzüstü (prone) yatış egzersizleri ile de desteklenmelidir. Tam ekstansiyon kazanılmadan, fleksiyon için zorlayıcı egzersizlere başlanmamalıdır, çünkü fleksiyonu pasif olarak agresif bir şekilde zorlamak dizdeki enflamatuvar cevabı artırarak mevcut durumu daha da olumsuzlaştırabilir. Uygun şekilde yapılan fizik tedaviye rağmen yeterli düzelme sağlanamayan olgularda konservatif tedavide çok ısrarcı olunmamalı ve cerrahi tedavi gündeme gelmelidir.

\section{TEKNIK FAKTÖRLER}

ÖÇB yaralanması sonrası oluşabilecek komplikasyonlardan sorumlu önemli faktörlerden biri de cerrahi teknikte yapılan hatalardır. Greftin yanlış yerleştirilmesi ameliyat sonrası dönemde karşılaşılan diz hareket kısıtlılığının önemli nedenlerindendir (Şekil 2). Greftin tibial bölümünü anteriora yerleştirmek noçta sıkışma yapacağından ekstansiyon kusuru oluşturacaktır. ${ }^{[10]}$ Ek olarak bu durumda greftin üzerine aşırı tensil kurvet yüklenmesi greft yetmezliği riskini artıracaktır. ${ }^{[10-12]}$ Dizde ameliyat sonrası fleksiyon kısıtlıığına yol açabilecek başka bir teknik hata da femoral tüneli fazlaca anteriorda açmak olacaktır. Tibial tüneli olması gereken yerin daha posteriorunda açmak ise tünelin vertikal yerleşimli olmasına, dolayısıyla rotasyonel stabilitenin kaybolmasına, arka çapraz bağ impingement'ına ve greftin erken dönemde yetmezliğine neden olacaktır. ${ }^{[11]}$

Greft tespitinin kaç derecede yapılacağı ve bunun ameliyat sonrası diz hareket açıklığına nasıl yansıyacağı literatürde hala tartışıımaktadır. Nabors ve ark.'nın biyomekanik çalışmasında tam ekstansiyonda yapılacak greft tespitinin dizde EHA kaybına neden olmayacağı bildirilirken ${ }^{[13]}$, bunu destekleyen başka bir çalışmada ise Lubowitz tam ekstansiyonda tespit edilecek greftin aşırı gerilmeye maruz kalmayacağını ve dolayısıyla daha az rüptür riski olacağını belirtmiştir. ${ }^{[14]}$ Ancak Debandi ve ark.'nın yaptığı biyomekanik çalışmada optimal tespit derecesinin $30^{\circ}$ semifleksiyon olduğu bildirilmektedir ${ }^{[15]}$, bu çalışmada dizin pivot shift testine en iyi $30^{\circ}$ semifleksiyonda karşı koyduğu ve bu tespit açısının dizin rotasyonel biyomekaniğine daha uygun olduğu vurgulanmaktadır.

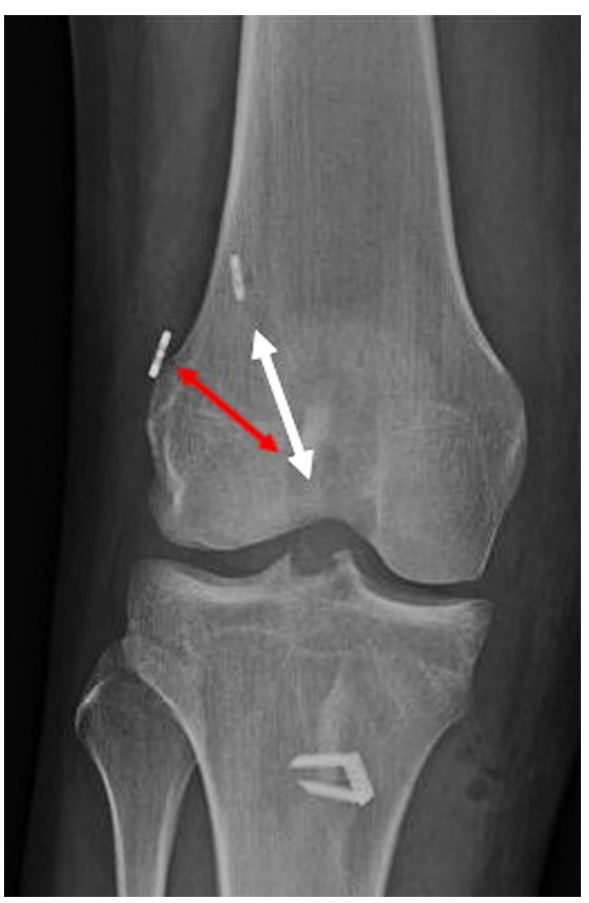

Şekil 2. Yirmi iki yaşında profesyonel voleybolcu kadın hasta, iki ay önce hamstring tendonu ile ÖÇB rekonstrüksiyonu. Ameliyat sonrası hareket kısıtlılığı ile başvurdu (EHA 0-80²). Sağ diz AP grafide femoral tünelin ne kadar vertikal olduğu görülüyor (beyaz ok). Ameliyat sonrası 3. ayda kontralateral hamstring ile ÖÇB revizyonu. Revizyon ameliyatındaki femoral tünel görülüyor (kırmızı ok).

Cerrahi sırasında dikkat edilmesi gereken bir diğer konu da greft alınması sonrasında, o bölgede oluşabilecek hematomdur. Donör sahada çalışırken pes anserinus bölgesi disseksiyonu sırasında titiz çalışmak önemlidir. Greft alınan bölgede oluşan hematomun, ilerleyen günlerde distale migre olması rehabilitasyonu olumsuz etkileyebilir. Donör sahaya dren konulması bunu önleme adına basit ve etkili bir yöntemdir. Drene rağmen oluşan ve sebat eden inatçı hematomlarda enerjik davranıp hematomu drene etmek rehabilitasyonu aksatmamak adına önemlidir.

\section{AMELIYAT SONRASI DÖNEM VE REHABILITASYON}

ÖÇB cerrahisi sonrası dizin immobilizasyonundan olabildiğince kaçınmak konusunda bir fikir birliği (konsensus) vardır. Erken hareket ağrıyı azaltır, kıkırdağın beslenmesini sağlar, skar dokusu gelişmesini ve dolayısı ile artrofibrozisi engeller. ${ }^{[16]}$ Ameliyat sonrası erken dönemde diz EHA egzersizlerinin yanında kuadriseps kontrol egzersizleri ve patella mobilizasyonu da oluşabilecek infrapatellar kontraktürleri engellemek adına önemlidir. 


\section{ENFEKSIYON}

ÖÇB cerrahisi sonrası eklem enfeksiyonu nadir ama ciddi ve tedavisi uzun süren bir komplikasyondur $(\% 0,14-1,71) .{ }^{[17]}$ Matava ve ark.'nın 61 ortopedik cerrah ile yaptığı çalışmada ameliyat sonrası gelişen septik artritte tedavi tercihleri incelenmiştir. ${ }^{[18]} \mathrm{Bu}$ çalışmada tedaviye karar vermede eklemde mikroorganizmanın üretilmesi ve sepsis belirtilerinin şiddeti belirleyici olarak yorumlanırken, cerrahların ortak tedavi tercihinin kültür spesifik parenteral antibiyoterapi ile birlikte artroskopik debridman ve irrigasyon olduğu vurgulanmıştır. Debridman sırasında ÖÇB greftinin olabildiğince korunması ilk tercihtir, ancak ÖÇB cerrahisi sonrası hedef stabil diz elde etmek olmakla birlikte kronik dirençli enfeksiyonlarda grefti feda etmek dizin geleceği açısından hayati bir önem taşımaktadır.

Bu korkulan komplikasyonu önlemek adına yapılan birçok çalışma vardır, örneğin Schuster ve ark., grefti dizin içine yerleştirmeden vankomisinli solüsyona tabi tutmuşlar ve 2294 olguda hiç enfeksiyona rastlanılmadığını bildirmişlerdir. [19]

Vankomisin kullanımı ilk olarak 2012 yılında Vertullo ve ark. tarafindan tarif edilmiştir. Bu geriye dönük çalışmada vankomisin emdirilmiş olgularda enfeksiyon oranının daha düşük olduğunu bildirilmektedir. ${ }^{[20]}$

Özetle enfeksiyon ÖÇB cerrahisi sonrası oluşabilecek ve sonuçları yıkıcı (katastrofik) olabilen bir komplikasyondur. Enfeksiyon riskini minimuma indirmek için genel kabul görmüş hususları şu şekilde özetlemek mümkündür:

- Önceki enfeksiyon hikâyesini iyi değerlendirmek (MRSA hikâyesi).

- Cildin durumu: ciltte ekimoz, bül olması, cerrahi alandaki kılların iyi temizliği ve steril örtümden önce batikonlu firçalar ile yıkanması.

- Cerrahi öncesi profilaktik antibiyotik verilmesi.

- Greft alınırken olabildiğince keskin disseksiyon yapmak, künt parmak disseksiyonundan kaçınmak, greft alınan alanda kanama kontrolü yapmak ve greft alındıktan sonra dren koymak.

- Ameliyat sonrası gelişebilecek hematom riskini azaltmak için eklem içinde çalışırken minör kanama odaklarını koterize etmek.

- Cerrahi malzemelerin ameliyathane personeli ve hemşire tarafından her zaman çift kontrolden geçirilmesi.

- Ameliyat sonrası oluşabilen hematomun yakın takibi, gerekirse ponksiyonu. Yalnız sık ponksiyonun da enfeksiyon ihtimalini artırabileceği unutulmamalıdır.

- Tanıda ameliyat sonrası septik artrit düşünüldügünde artroskopik debridman ve irrigasyon konusunda çabuk davranmak ve gerekirse tekrardan kaçınmamak.
ÖÇB rekonstrüksiyonu günümüzde artık çok sık uygulanan ve başarı oranının yüksek olduğu bir cerrahidir. Ancak ortaya çıkan komplikasyonların tedavisi oldukça güç olabilmektedir. Bu yüzden risk faktörlerini minimalize etmek, komplikasyon ortaya çıktığında da enerjik davranmak çok önemlidir.

\section{KAYNAKLAR}

1. Noyes FR. Knee Disorders, Surgery, Rehabilitation, Clinical Outcomes. Saunders Elsevier; 2009.

2. Salzler JM, Lin A, Miller CD, Herold S, Irrgang JJ, Harner CD. Complications after arthroscopic knee surgery. The Am J Sports Med 2014;42(2):292-6. Crossref

3. Wasilewski SA, Covall, DJ, Cohen S. Effect of surgical timing on recovery and associated injuries after anterior cruciate ligament reconstruction. Am J Sports Med 1993;21(3):33842. Crossref

4. Shelbourne KD, Patel DV, Martini DJ. Classification and management of arthrofibrosis of the knee after anterior cruciate ligament reconstruction. Am J Sports Med 1996;24(6):857-62. Crossref

5. Harner CD, Irrgang JJ, Paul J, Dearwater S, Fu FH. Loss of motion after anterior cruciate ligament reconstruction. Am J Sports Med 1992;20(5):499-506. Crossref

6. Bach BR, Jones GT, Sweet FA, Hager CA. Arthroscopyassisted anterior cruciate ligament reconstruction using patellar tendon substitution. Two- to four-year follow-up results. Am J Sports Med 1994;22(6):758-67. Crossref

7. Bosch U, Zeichen J, Lobenhoffer P, Albers I, van Griensven, M. Arthrofibrose. Arthroskopie 1999;12(3):117-20. Crossref

8. Lawrance SE, Shelbourne KD. Treatment and Rehabilitation of Arthrofibrosis of the Knee (Chap. 52). In: Giangarra CE, Manske RC, editors. Clinical Orthopaedic Rehabilitation: A Team Approach, 4th ed. Elsevier; 2017. p. 353-8.

9. Arsoy D, Salib CG, Trousdale WH, Tibbo ME, Limberg AK, Viste A, Lewallen EA, Reina N, Yaszemski MJ, Berry DJ, van Wijnen AJ, Morrey ME, Sotelo JS, Abdel MP. Joint Contracture Is Reduced by Intra-Articular Implantation of RosiglitazoneLoaded Hydrogels in a Rabbit Model of Arthrofibrosis. J Orthop Res 2018;36(11):2949-55. Crossref

10. Howell SM, Clark JA. Tibial tunnel placement in anterior cruciate ligament reconstructions and graft impingement. Clin Orthop Relat Res 1992;(283):187-95. Crossref

11. Romano VM, Graf BK, Keene JS, Lange RH. Anterior cruciate ligament reconstruction. The effect of tibial tunnel placement on range of motion. Am J Sports Med 1993;21(3):415-8. Crossref

12. Tjoumakaris FP, Herz-Brown A, Bowers AL, Sennett BJ, Bernstein J. Complications in Brief: Anterior Cruciate Ligament Reconstruction. Clin Orthop Relat Res 2012;470(2):630-6. Crossref

13. Nabors ED, Richmond JC, Vannah WM, McConville OR. Anterior cruciate ligament graft tensioning in full extension. Am J Sports Med 1995;23(4):488-92. Crossref

14. Lubowitz JH. Anatomic ACL Reconstruction Produces Greater Graft Length Change During Knee Range-Of-Motion Than Transtibial Technique. Knee Surg Sports Traumatol Arthrosc 2014;22(5):1190-5. Crossref

15. Debandi A, Maeyema A, Hoshino Y, Asai S, Goto B, Smolinski $P$, Freddie F. The Influence of Knee Flexion Angle for Graft Fixation on Rotational Knee Stability During Anterior Cruciate Ligament Reconstruction: A Biomechanical Study. Arthroscopy 2016;32(11):2322-8. Crossref 
16. Rowe PJ, Myles CM, Walker C, Nutton R. Knee joint kinematics in gait and other functional activities measured using flexible electrogoniometry: how much knee motion is sufficient for normal daily life? Gait Posture 2000;12(2):143-55. Crossref

17. Judd D, Bottoni C, Kim D, Burke CPTM, Hooker MAJS. Infections following arthroscopic anterior cruciate ligament reconstruction. Arthroscopy 2006;22(4):375-84. Crossref

18. Matava MJ, Evans TA, Wright RW, Shively RA. Septic arthritis of the knee following anterior cruciate ligament reconstruction: results of a survey of sports medicine fellowship directors. Arthroscopy 1998;14(7):717-25. Crossref
19. Schuster $P$, Schlumberger $M$, Mayer $P$, Eichinger $M$, Geßlein $M$, Reddemann F, Richter J. Soaking of the graft in vancomycin dramatically reduces the incidence of postoperative septic arthritis after anterior cruciate ligament reconstruction. Knee Surg Sports Traumatol Arthrosc 2020. Crossref

20. Vertullo J, Quick M, Jones A, Greyson JE. A surgical technique using presoaked vancomycin hamstring grafts to decrease the risk of infection after anterior cruciate ligament reconstruction. Arthroscopy 2012;28(3):337-42. Crossref 